INVESTIGACIONES

\title{
Aula invertida mediada por el uso de plataformas virtuales: un estudio de caso en la formación de profesores de física
}

\author{
Flipped classroom mediated by the use of virtual platforms: a case study of pre-service \\ teacher education in physics
}

\author{
Carla Hernández-Silva ${ }^{a}$, Silvia Tecpan Flores ${ }^{b}$ \\ ${ }^{a}$ Departamento de Física, Facultad de Ciencia, Universidad de Santiago de Chile \\ Correo electrónico: carla.hernandez.s@ usach.cl \\ ${ }^{\text {b }}$ Departamento de Física, Facultad de Ciencia, Universidad de Santiago de Chile \\ Correo electrónico: silvia.tecpan@usach.cl
}

\begin{abstract}
RESUMEN
Se presentan detalles y resultados de la implementación de un modelo de aula invertida en un curso de didáctica para futuros profesores de física en una universidad estatal de Chile, utilizando una plataforma virtual de acceso libre. A través de un estudio de caso con enfoque de investigación cualitativo descriptivo, se identificaron diversas ventajas y desventajas respecto al aula invertida como estrategia de instrucción, declaradas por los 31 participantes al término del semestre. Entre ellas, se destaca el desarrollo de hábitos y autorregulación para el aprendizaje, lo que contrasta por otra parte, con la dificultad adicional que supone la constante necesidad de revisar los materiales previos a la clase disponibles en la plataforma virtual seleccionada como apoyo para la docencia. Se discuten implicaciones para la formación del profesorado y sugerencias para futuras implementaciones.
\end{abstract}

Palabras clave: Aprendizaje colaborativo, Didáctica, Entorno virtual, TIC.

\begin{abstract}
This article shows the main results of the implementation of a flipped classroom model by using a free access virtual platform in a didactic course for future physics teachers at a state university in Chile. Through a case study with a qualitative-descriptive research approach, at the end of the semester the 31 participants identified several advantages and disadvantages regarding the flipped classroom as an instructional strategy. Among them, the development of habits and self-regulation for learning are highlighted, which contrasts with the additional difficulty of constantly revising the pre-class materials available on the virtual platform selected as support for teaching. Implications for teacher training and suggestions for future implementations are discussed.
\end{abstract}

Key words: Collaborative learning, Didactic, Virtual environment, ICT. 
Estudios Pedagógicos XLIII, N 3: 193-204, 2017

AULA INVERTIDA MEDIADA POR EL USO DE PLATAFORMAS VIRTUALES: UN ESTUDIO DE CASO EN LA FORMACIÓN DE PROFESORES DE FÍSICA

\section{INTRODUCCIÓN}

Los nuevos paradigmas educativos apuntan a una enseñanza centrada en el estudiante, donde se fomente la colaboración entre pares, la participación activa y el desarrollo de habilidades comunicativas y de razonamiento de orden superior (Mestre, 2001). Esto supone la necesidad de que la formación del profesorado se ajuste a los nuevos requerimientos, no obstante, aún predominan los estilos magistrales de clases expositivas donde el estudiante tiene un rol mayormente pasivo (Gaete, 2011). Los modelos educativos comúnmente llamados tradicionales, son caracterizados por estar centrados en el contenido y en el rol protagónico del docente dentro del aula, promoviendo actividades que se enfocan a memorizar, retener, conocer y comprender contenidos de manera individual. Estas habilidades se corresponden con los niveles más bajos de trabajo cognitivo según la taxonomía propuesta por Bloom (1964). En consecuencia, el espacio de interacción que supone el aula no es aprovechado para actividades que potencien el desarrollo de habilidades más complejas, tales como aplicar, analizar, evaluar, crear, y en general, aquellas que pueden realizarse en ambientes colaborativos fomentando el diálogo entre pares.

Ahora bien, independiente del enfoque o paradigma que prime en el aula, no se puede desconocer el impacto que han tenido las tecnologías digitales en distintos ámbitos de la educación, provocando cambios desde las estrategias de enseñanza-aprendizaje hasta el currículum y tanto en el rol de los estudiantes como de los docentes (Pérez, Romero y Romeu, 2014). De acuerdo con Zainuddin \& Halili (2016) las tecnologías de información y comunicación (TIC) proveen de acceso instantáneo a la información a través de distintos dispositivos tecnológicos con acceso a internet.

En este marco, surge como alternativa el modelo llamado Aula Invertida también conocido como Flipped Classroom (Lage, Platt \& Treglia, 2000), que propone tal y como su nombre sugiere, invertir las actividades realizadas habitualmente en el aula para dar paso a otras que favorezcan el aprendizaje en entornos colaborativos (Collazos y Mendoza, 2006). Según Carvalho \& McCandless (2014) lo que era tradicionalmente hecho en la sala de clases, como la exposición de contenido, es llevado fuera de ella como una actividad previa a la clase, y del mismo modo, las actividades que normalmente se daban como tareas para hacer en casa o fuera del aula, ahora se convierten en actividades de participación activa dentro de la clase. Esta decisión implica que las actividades que se realizan en casa son las que se ubican ahora en los niveles más bajos de la taxonomía de Bloom, como se observa en la figura 1, mientras que aquellas que requieren de mayor esfuerzo cognitivo se pueden realizar en la sala de clases con la orientación del profesor (Zainuddin \& Halili, 2016).

Para realizar clases con un modelo de aula invertida, es necesario utilizar herramientas que faciliten el intercambio de información entre el docente y los estudiantes tanto dentro como fuera del aula, y para el desarrollo o preparación previa de las clases. En este sentido, el uso de las TIC representa una excelente oportunidad, que favorece además el desarrollo de habilidades digitales necesarias para la sociedad del conocimiento (Martínez, Esquivel y Castillo, 2014).

\subsection{ANTECEDENTES TEÓRICOS}

El objetivo de utilizar las TIC debe responder a la necesidad de mejorar la interacción entre estudiantes y docentes, tanto dentro como fuera del aula, y de propiciar oportunidades de 


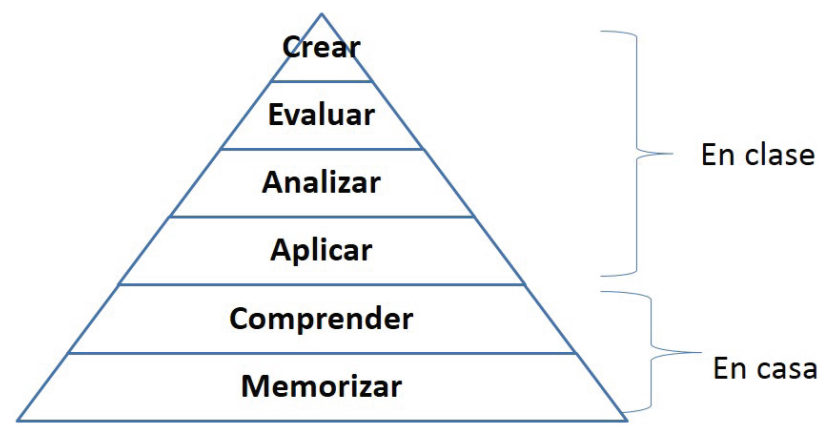

Figura 1. Taxonomía de Bloom en el aula invertida (Adaptado de Zainuddin \& Halili, 2016).

aprendizaje colaborativo, sobre todo cuando las clases mantienen su modalidad presencial (Ibáñez, 2004). En este sentido, la integración de las tecnologías en los procesos formativos no sólo contribuyen a mejorar la calidad de la docencia, sino también a fomentar la regulación de los aprendizajes por parte de los estudiantes (Conde, 2004).

Riascos, Quintero y Ávila (2009) exponen una interesante comparación entre un ambiente de aprendizaje tradicional y uno que incorpora las TIC, destacando el pensamiento crítico y la toma de decisiones informadas, que si bien no son exclusivas de los nuevos ambientes de aprendizaje se potencian al exponer a los estudiantes a más fuentes de información en donde deben discriminar su veracidad. Además, los autores reconocen que en el ambiente tradicional el trabajo del estudiante suele ser individual y pasivo, mientras que en el ambiente con TIC se potencia el trabajo colaborativo que propicia un aprendizaje activo. Se entiende el aprendizaje activo como aquel en que los estudiantes están completamente implicados, tanto física como mentalmente (Karamustafaoglu, 2009), y que se refuerza con la interacción constante entre los integrantes de los grupos.

En esta línea, el uso de plataformas virtuales dentro de un modelo de aula invertida puede facilitar el acceso a contenidos seleccionados por el docente, para su uso antes, durante y después de clases, o como espacio colaborativo para facilitar la interacción entre estudiantes y profesores fuera de la clase, a través del espacio virtual. Zainuddin \& Halili (2016) encontraron que entre las plataformas más utilizadas en aula invertida están: un blog como plataforma en línea para compartir las videoconferencias; WebQuest para establecer el aprendizaje activo de los estudiantes en la clase, televisión interactiva en distintos campus, conferencias de video en YouTube, colaboración en Google Docs y Google Hangout, Blackboard®, entre otros.

Ahora bien, utilizar plataformas virtuales propicia la implementación del aula invertida, sin que ello implique transformar un curso presencial en uno virtual. Platero, Tejeiro y Reis (2015) indican que el aula invertida como metodología y herramienta de aprendizaje, invierte el modo de enseñanza tradicional provocando que gran parte de la obtención de conocimiento se produzca mediante el aprendizaje autónomo con el estudio fuera del aula y que la realización de tareas y prácticas se hagan en el aula con el apoyo de los compañeros y el docente, transfiriendo el control del aprendizaje al estudiante. Bergmann \& Sams (2012) fueron quienes popularizaron el término, así como también el uso de videos 
para grabar clases que sirvieran de apoyo, sin embargo, es preciso destacar que se trata del uso de tecnología en general, no solo videos como medio para acceder al material de apoyo fuera del aula. Diversos autores han propuesto adaptaciones al modelo de aula invertida, amoldando diferentes estrategias a las realidades de cada aula (Bergmann \& Sams, 2014; Lage, Platt \& Treglia, 2000).

Si bien se tienen pocas evidencias de la aplicación de aula invertida en la formación del profesorado (Sadaghiani, 2012; Zainuddin \& Halili, 2016) se considera un modelo adecuado para los cursos de formación docente ya que fomenta la responsabilidad del estudiante sobre su propio aprendizaje, y al mismo tiempo libera el tiempo de clase para exponer a los maestros en formación a una variedad de estrategias de instrucción que favorecen el aprendizaje del estudiante durante la aplicación de los contenidos que han aprendido (West \& Graham, 2007).

Aunque aún existe poca investigación sobre el rendimiento de los estudiantes al utilizar aula invertida, sí se han reportado beneficios tales como el compromiso que desarrollan con su propio aprendizaje y el aumento de la retroalimentación del estudiante-profesor (Goodwin \& Miller, 2013; Sadaghiani, 2012).

A medida que crece la brecha entre la pedagogía tradicional y las necesidades de los estudiantes "conectados" (Prensky, 2008), los programas de formación docente deben adaptarse a este escenario ya que los nuevos maestros basan su práctica profesional en gran medida del conocimiento y las habilidades aprendidas en su formación inicial (West \& Graham, 2007).

Considerando los antecedentes y lineamientos teóricos anteriormente expuestos, esta investigación tuvo como objetivo describir tanto la implementación como las ventajas y desventajas que futuros profesores de física identifican para el aula invertida como modelo de enseñanza y aprendizaje, después de haber participado de un curso de didáctica de la física realizado completamente con este modelo.

\section{MATERIAL Y MÉTODOS}

Se realizó un estudio de caso con enfoque descriptivo y metodología cualitativa (Merriam, 2009), lo que se corresponde con otros estudios previamente reportados (Platero, Tejeiro, y Reis, 2015; Zainuddin \& Halili, 2016). Se utilizó un diseño por encuesta que tuvo como instrumento una guía de entrevista que se realizó de manera grupal (Giroux y Tremblay, 2004). Se aplicó muestreo no probabilístico a juicio con lo que la muestra quedó constituida por 31 estudiantes en el marco de la finalización de un curso de didáctica, de un semestre de duración, que se imparte en la carrera de pedagogía en física de una universidad estatal chilena.

Tal y como se sugiere en la literatura, en una versión anterior del mismo curso se realizó una aplicación del aula invertida parcial y paulatina, en algunos temas (Observatorio de Innovación Educativa, 2014; Platero, Tejeiro y Reis, 2015). Esto permitió seleccionar y conocer con anticipación la plataforma tecnológica de apoyo y las estrategias didácticas a utilizar tanto dentro como fuera del aula, evaluando resultados que permitieron modificar el modelo antes de llegar a implementarlo al curso completo.

En función de los resultados de la implementación previa, se diseñó un modelo de aula invertida que favoreció el trabajo colaborativo en clases, tanto en pequeños grupos como en plenario, a través de diversas estrategias de aprendizaje activo. 
Para el estudio fuera del aula y la preparación previa de clases, se realizó una selección de material relacionado con los contenidos del curso de didáctica mencionado que incluían videos disponibles en Youtube, artículos de revistas científicas, capítulos de libros y test estandarizados, entre otros. Los estudiantes podían ver los videos o materiales de estudio en varias ocasiones, reflexionar sobre algunas de las preguntas planteadas, y llegar a clase con sus propias inquietudes. A modo de contraste, en un aula tradicional los estudiantes a menudo están todavía procesando el contenido de la lección cuando la clase ya demanda la aplicación de ese contenido sin que lo hayan comprendido plenamente. Para lograr que los estudiantes estén más comprometidos y activos en su aprendizaje, como objetivo propio del uso de este modelo (Goodwin \& Miller, 2013), es necesario reestructurar las actividades de clase para crear experiencias de aprendizaje profundo.

La figura 2 resume las características del modelo de aula invertida implementado, donde se aprecian las distintas modalidades de participación y el espacio en que se realizan.

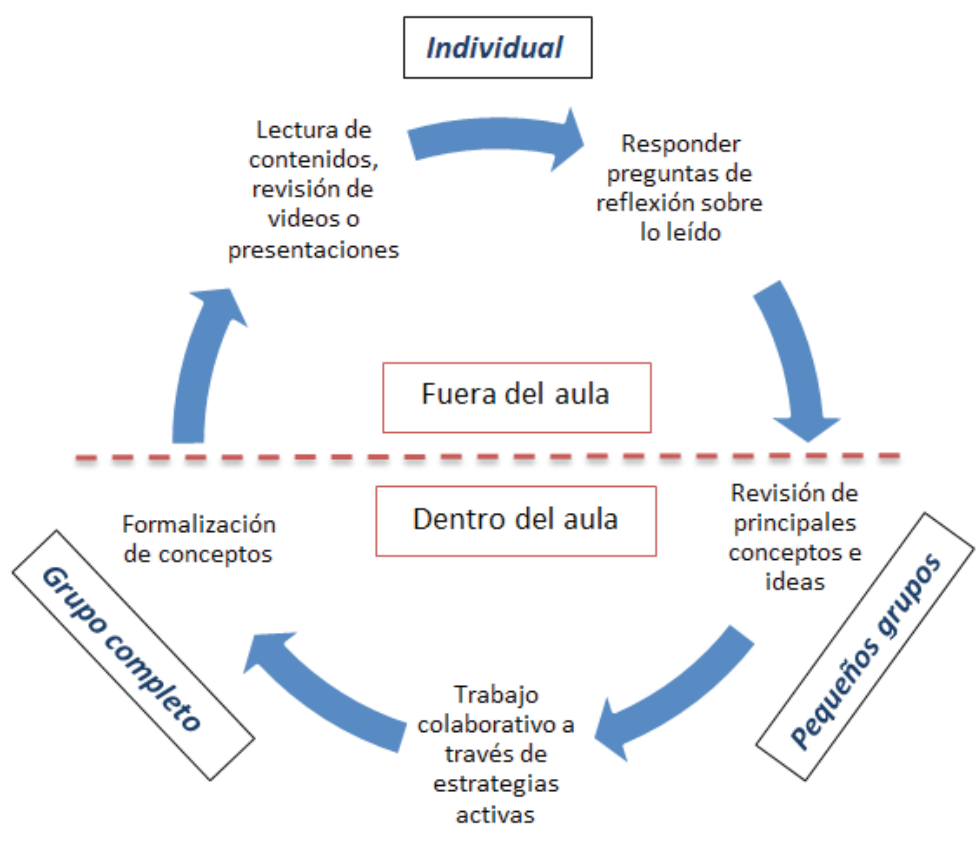

Figura 2. Modelo de aula invertida para el curso de didáctica de la física (Elaboración propia).

La plataforma virtual escogida para apoyar la clase y albergar los recursos didácticos seleccionados fue "Google Classroom" ya que todos los estudiantes cuentan con un correo electrónico institucional alojado en Google que facilita el acceso gratuito y permanente a la plataforma. Por otra parte, su formato es similar a un blog que permite alojar todo tipo de documentos y recursos interactivos, además de generar tareas con plazos fijos, y publicaciones diversas de manera periódica, tanto por parte del docente como de los estudiantes. 


\subsection{DETALLES Y CONTEXTO DE LA IMPLEMENTACIÓN}

El curso de didáctica de la física donde se implementó el modelo de aula invertida consta de dos grandes módulos. Un primer módulo donde se enseña contenido teórico respecto a las bases didácticas de la enseñanza de la física y un segundo donde se espera que los estudiantes apliquen lo aprendido planificando e implementando una clase de física con estrategias de aprendizaje activo.

Dentro de los contenidos teóricos que se incluyen en el curso, los principales son: a)Teorías de aprendizaje y evolución de paradigmas educativos, b) Ideas previas, preconceptos y cambio conceptual, c) Teoría del aprendizaje significativo, d) Rol de las preguntas en la enseñanza de la física, e) Ciclos de aprendizaje y f) Estrategias de aprendizaje activo para física.

Para cada uno de los contenidos, el docente preparó previamente material que fue compartido antes de cada clase a través de la plataforma y, en algunos casos, se plantearon preguntas para reflexionar respecto a los temas que pudieron ser respondidas por los estudiantes en línea de manera anticipada a la sesión presencial. Durante el desarrollo del curso, los estudiantes pudieron interactuar con la plataforma tanto para obtener información complementaria, como también para publicar material por ellos creado y comunicarse con el profesor. Además, la plataforma permitía que los estudiantes fuesen enviando sus tareas, facilitando al docente el acceso a la mismas, la revisión y calificación en línea.

Para los estudiantes, acceder al material seleccionado por el docente, fuera del aula, posibilitaba llegar a clases con conocimiento previo del contenido a abordar y con inquietudes que podían ser planteadas durante el desarrollo de la misma.

Desde el punto de vista del docente, el hecho de que sus estudiantes conocieran previamente los contenidos básicos a abordar, permitió que pudiera destinar la mayor parte de la clase a realizar actividades que favorezcan la discusión y trabajo entre pares, analizando el contenido y resolviendo inquietudes que surgen de la aplicación y uso que hacen del él. La tabla 1 resume la diversidad de actividades realizadas por los estudiantes a lo largo del semestre, en el caso descrito del curso de didáctica.

Tabla 1. Actividades realizadas por los estudiantes dentro y fuera del aula con el modelo de aula invertida implementado en el curso de didáctica

\begin{tabular}{|l|l|}
\hline $\begin{array}{l}\text { Dentro del Aula } \\
\text { (en grupos colaborativos) }\end{array}$ & $\begin{array}{l}\text { Fuera del Aula } \\
\text { (individual o en grupos pequeños) }\end{array}$ \\
\hline Construir tablas comparativas & Responder test \\
\hline Elaborar mapas conceptuales y redes de conceptos & Ver y analizar videos \\
\hline Analizar libros de texto escolares & Leer textos científicos \\
\hline Consensuar definiciones & Responder preguntas orientadoras \\
\hline Crear recursos didácticos & Generar apuntes y resúmenes \\
\hline Debatir, dialogar y preguntar de manera reflexiva & Compartir y publicar material generado en clases \\
\hline Diseñar y planificar clases & \\
\hline Implementar estrategias activas & \\
\hline Evaluar entre pares & \\
\hline
\end{tabular}


De acuerdo con Lim, Kim, Lee, Kim \& Han (2014) una característica del modelo de aula invertida es que permite idear y gestionar distintos tipos de aula dependiendo de la experiencia del docente, los estilos de aprendizaje de los estudiantes y los recursos disponibles, lo que coincide con los hallazgos de Zainuddin \& Halili (2016) quienes indican que el aula invertida favorece la integración y sincronización con los estilos de aprendizaje de los estudiantes al proponer diversas estrategias didácticas en línea y presenciales lo que permite un aprendizaje activo eficaz y el desarrollo de habilidades de pensamiento de orden superior.

Más allá de la atención a la diversidad de los estilos de aprendizaje, los contenidos específicos a abordar también determinan el tipo de estrategias didácticas a realizar dentro de este modelo (Observatorio de Innovación Educativa, 2014). Por ejemplo, en una asignatura de ciencia se sumarían las actividades propias de la experimentación, en matemáticas se agregaría la resolución de problemas, en arte consideraríamos el trabajo manual, etc..., todas ellas actividades que habitualmente son realizadas por los estudiantes como tareas a realizar después de una clase.

\section{RESULTADOS}

Al finalizar el curso, se realizó una entrevista grupal con todos los estudiantes para conocer sus opiniones respecto al modelo de aula invertida implementado, el cual era desconocido para ellos hasta entonces.

A continuación se presentan algunas frases extraídas de manera literal de la transcripción realizada, donde se da cuenta de diferentes aspectos en los cuales los estudiantes reflexionaron. Por ejemplo, en algunos casos, se hizo alusión a lo novedoso del modelo desde un punto de vista de profesor y alumno, a pesar de que no se dio esa instrucción en la entrevista grupal:

Alumno 9: "La idea de aula invertida es un concepto nuevo y muy interesante para mí, tanto como alumno como profesor, debido a que mi concepto de clase estaba asociado a las clases tradicionales, ya que mi experiencia fue así en el colegio".

Es interesante el nivel de reflexión obtenido respecto a la propia formación como estudiantes de pedagogía:

Alumno 4: “[...] el curso me permitió observar aún muchas deficiencias que poseo como futuro educador, como abordarlas y también nuevas herramientas aplicables en las salas de clases."

Por otra parte, se discutió respecto a los propios cambios que tuvieron que hacer como estudiantes, en cuanto a los hábitos y la gestión del tiempo de dedicación durante el desarrollo del curso:

Alumno 15: "En un principio no me gustaba, porque requería dedicarle más tiempo revisando la plataforma y viendo los textos que mandaban, lo que me daba pereza hacer. Después de un tiempo me acostumbré y fue más fácil" 
Estudios Pedagógicos XLIII, N 3: 193-204, 2017

AULA INVERTIDA MEDIADA POR EL USO DE PLATAFORMAS VIRTUALES: UN ESTUDIO DE CASO EN LA FORMACIÓN DE PROFESORES DE FÍSICA

En otros casos, hubo algunos comentarios relacionados con posturas críticas respecto a la implementación del aula invertida, que iban más allá del simple gusto personal.

Alumno 23: "Como crítica, la principal falla que puedo ver es que depende mucho del tiempo fuera de clases del estudiante, lo cual se hace difícil por el hecho de tener otras asignaturas, las cuales también dependen de este tiempo del estudiante."

En términos generales, se lograron identificar a partir del diálogo establecido en la entrevista grupal, un total de 76 nociones expresadas como unidades de significado por la totalidad de los estudiantes, las cuales se organizaron con base en categorías incipientes. En la tabla II se presentan 49 de las nociones, contabilizadas en función de la frecuencia de aparición, que pudieron ser clasificadas como ventajas y desventajas en torno al uso del modelo de aula invertida. Se distinguen entre aquellas planteadas desde una perspectiva de estudiante, y otras en que se posicionan como profesores, a pesar de que no se dio esa instrucción explícitamente durante la entrevista.

Tabla 2. Nociones de futuros profesores de física sobre modelo de aula invertida implementado

\begin{tabular}{|c|c|c|c|}
\hline \multirow{2}{*}{ Perspectiva } & \multirow{2}{*}{ Noción } & \multicolumn{2}{|c|}{ Frecuencia (f) } \\
\hline & & Ventajas & Desventajas \\
\hline \multirow{8}{*}{ Estudiante } & Requiere y promueve hábitos de estudio & 9 & 1 \\
\hline & $\begin{array}{l}\text { Requiere responsabilidad en la preparación de clases / } \\
\text { genera expectativa }\end{array}$ & 5 & 1 \\
\hline & Favorece la reflexión sobre el aprendizaje & 5 & - \\
\hline & Favorece la autorregulación/requiere autonomía & 5 & - \\
\hline & $\begin{array}{l}\text { Requiere mayor dedicación, tiempo y compromiso al } \\
\text { estudio del contenido ya que no está restringido al } \\
\text { tiempo de clase }\end{array}$ & 4 & 2 \\
\hline & $\begin{array}{l}\text { La dedicación fuera del aula es útil y necesaria para el } \\
\text { trabajo dentro del aula, cosa que no necesariamente } \\
\text { ocurre en un modelo tradicional }\end{array}$ & 3 & - \\
\hline & $\begin{array}{l}\text { Favorece el trabajo en equipo y respeto por las ideas } \\
\text { de otros/ Favorece el aprendizaje colaborativo }\end{array}$ & 3 & - \\
\hline & El trabajo fuera de clase sigue siendo una tarea $(*)$ & - & 1 \\
\hline \multirow{6}{*}{ Profesor } & Será útil para cuando sea profesor & 3 & \\
\hline & Propicia el rol activo y protagónico del estudiante & 3 & - \\
\hline & Adecuado a distintos estilos de aprendizaje & 1 & - \\
\hline & $\begin{array}{l}\text { Uso parcial del aula invertida, no para todo el curso, } \\
\text { buscar uso equilibrado }\end{array}$ & - & 1 \\
\hline & $\begin{array}{l}\text { La estrategia sirve sólo para algunos contenidos que } \\
\text { no sean muy complejos }(*)\end{array}$ & - & 1 \\
\hline & El aula invertida permite extender el horario de clases $(*)$ & 1 & - \\
\hline
\end{tabular}


Las respuestas señaladas con asterisco (*) se han clasificado como nociones erróneas, ya que no se corresponden con lo reportado en la literatura especializada.

El interés e importancia que suponen los resultados obtenidos, a pesar de ser un estudio de caso aplicado a una muestra pequeña, radica en considerar que el curso se implementó para profesores en formación, quienes a futuro podrán implementar en sus aulas el tipo de estrategias, herramientas y conocimiento adquirido en esta etapa. Sin embargo, si la formación del profesorado mantiene su estilo magistral y expositivo de clases, será muy difícil que las futuras generaciones de docentes logren innovar en el aula y enseñar en acuerdo a los paradigmas educativos actuales. Al respecto, cualquier cambio que se logre generar en ellos respecto a las formas de aprender y enseñar, puede implicar grandes diferencias en su rol profesional futuro.

\section{DISCUSIÓN Y CONCLUSIONES}

Predominan en las respuestas obtenidas las ventajas que aluden al desarrollo de hábitos de estudio y la autorregulación, lo cual puede deberse principalmente al hecho de que durante el curso los estudiantes pudieron aprender los contenidos de manera activa, asumiendo roles dentro de cada grupo que implicaba organizar información, coordinar diálogos y participaciones, entre otras actividades que requerían de autonomía y compromiso con el aprendizaje personal y del grupo. Este resultado concuerda con lo reportado por otros autores (Pierce \& Fox, 2012; Tune, Sturek \& Basile, 2013), respecto a que el aula invertida favorece el aprendizaje autónomo en los estudiantes y la autogestión.

En el caso de quienes reconocen la gran dedicación y responsabilidad que requiere estudiar bajo este modelo, la mayoría lo plantean como una ventaja mientras para un caso se asume como una dificultad, lo cual coincide con resultados de otros estudios que han reportado sobre la resistencia de algunos alumnos al trabajar por primera vez con el modelo de aula invertida (Lim et al., 2014; Observatorio de Innovación Educativa, 2014; Platero, Tejeiro y Reis, 2015).

Otro de los resultados identificados como ventajas para los estudiantes, respecto al uso de un aula invertida, es el reconocimiento hacia el tiempo que dedican para reflexionar sobre su propio aprendizaje, lo que coincide con lo reportado en otros estudios (Strayer, 2012).

Por otro lado, se ha identificado como noción errónea en las respuestas la consideración de futuros profesores respecto a que el tiempo fuera del aula es una extensión del horario de clases y por lo tanto, todo lo que no se alcanza a enseñar en el aula puede ser aprendido por el estudiante fuera de ella (Vaughan, 2014). Esta idea fuertemente arraigada, se contradice con las bases del aula invertida que como modelo educativo busca potenciar el aprendizaje a través de una mayor diversidad de actividades, lo que no implica una mayor cantidad de trabajo para el alumno (Observatorio de Innovación Educativa, 2014). Si bien se reconoce que el modelo de aula invertida es difícil de seguir por estudiantes de medio tiempo (Chen, Yang \& Hsiao, 2015), no es el caso del contexto de este estudio donde los estudiantes cuentan con becas que les permiten dedicación de tiempo completo. Sin embargo este aspecto, gestión del tiempo, debe ser considerado al momento de evaluar el contexto de implementación de aula invertida.

En relación con lo anterior, la noción de un estudiantes respecto a que todo el trabajo realizado fuera del aula cabe dentro de las llamadas "tareas", se contradice con las 
reflexiones del resto del grupo donde coinciden en la utilidad que tenía para el propio aprendizaje y trabajo en clases, el haber preparado previamente el contenido.

La noción respecto a que el modelo es válido solo para contenidos de menor complejidad, puede interpretarse como una fuerte dependencia o costumbre de los estudiantes hacia los modelos de aula tradicionales, donde se concibe que el aprendizaje y la enseñanza debe estar centrada en el contenido y que por ende, aquellos de mayor complejidad requieren del uso de estrategias conocidas y probadas. Esta noción puede surgir entre aquellos estudiantes que no han desarrollado estrategias adecuadas para el aprendizaje en línea como sugiere Al-Zahrani (2015).

\subsection{IMPLICANCIAS Y SUGERENCIAS}

Los resultados obtenidos aportan evidencia para continuar utilizando este modelo pedagógico en la formación inicial docente, pues les permitió reflexionar sobre los estudiantes con que se encontrarán en su vida profesional que se distinguen por estar habitualmente conectados. Lo anterior, les facilita el acceso a distintos contenidos de aprendizaje que si son correctamente gestionados por el docente contribuyen al aprendizaje profundo y reflexivo que la sociedad del conocimiento demanda. Como estudiantes también reflexionaron sobre el compromiso con su propio aprendizaje y la necesidad de desarrollar mejores hábitos de estudio.

En cuanto a las sugerencias para la implementación, cabe mencionar que no es necesario que el docente genere todo el material de manera original, pues es posible recurrir a recursos de excelente calidad en distintos sitios especializados tanto en aula invertida como en contenidos específicos de las distintas áreas de conocimiento, los cuales son compartidos por las comunidades de docentes a través de la red de manera gratuita. De este modo es posible utilizar materiales de diversos formatos siendo importante respetar y trasparentar la autoría en cada caso.

La inmersión gradual al modelo también es un aspecto a considerar pues se requiere la experticia del docente tanto en el uso de la plataforma como en las interacciones en los distintos espacios de aprendizaje. Al respecto, se sugiere comenzar implementando actividades con aula invertida para algunos temas o unidades del curso, e ir combinando con clases tradicionales para que los estudiantes también tengan la posibilidad de adaptarse.

Por otra parte, ante las nociones consideradas como érroneas que se detectaron, es importante discutir con mayor detalle la gestión del tiempo dentro del modelo teniendo en cuenta que en un aula tradicional siempre se requiere de tiempo de trabajo no presencial y autónomo por parte del estudiante, de modo que no se aumenta el tiempo de trabajo sino que se invierte para privilegiar la discusión y colaboración.

Finalmente, se considera importante recalcar que realizar un curso completamente con modelo de aula invertida implica aprovechar al máximo el potencial de las TIC en la educación, sobre todo con el uso de plataformas virtuales que favorece la interacción fuera del aula, sin perder las interacciones propias del aula presencial. 


\section{REFERENCIAS BIBLIOGRÁFICAS}

Al-Zahrani, A. (2015). From passive to active: The impact of the flipped classroom through social learning platforms on higher education students' creative thinking. Brit. J. Educ. Technol., 46(6), 1133-1148. doi: http://dx.doi.org/10.1111/bjet.12353

Bergmann, J., \& Sams, A. (2014). Flipped learning: Maximizing face time. $T+D, 68(2), 28-31$. Recuperado el 04 de octubre de 2016 desde goo.gl/NTUPvu

Bloom, B. S. (1964). Taxonomy of educational objectives (Vol. 2). New York: Longmans, Green.

Carvalho, H. \& Mc Candless, M. (2014). Implementing the flipped classroom. Revista HUPE-Rio de Janeiro, 13 (4), 39-45. Recuperado el 23 de noviembre de 2016 desde goo.gl/mqp7UF

Chen, S., Yang, S. \& Hsiao, C. (2015). Exploring student perceptions, learning outcome and gender differences in a flipped mathematics course, Brit. J. Educ. Technol., 47(6), 1096-112 doi: http:// dx.doi.org/10.1111/bjet.12278

Collazos, C. A., y Mendoza, J. (2006). Cómo aprovechar el aprendizaje colaborativo en el aula. Educación y educadores, 9(2), 61-76. Recuperado el 17 de diciembre de 2016 desde goo.gl/rTIDZv

Conde, C. S. (2004). Formación universitaria y TIC: nuevos usos y nuevos roles. RUSC. Universities and Knowledge Society Journal, 1(1), 1. Recuperado el 20 de diciembre de 2016 desde goo. $\mathrm{gl} / 28 \mathrm{BDQK}$

Giroux, S., y Tremblay, G. (2004). Metodología de las ciencias humanas. Distrito Federal, México: Fondo de Cultura Económica.

Gaete, R. A. (2011). El juego de roles como estrategia de evaluación de aprendizajes universitarios. Educación y educadores, 14(2), 289-307. Recuperado el 04 de noviembre de 2016 desde goo. gl/9673WN

Goodwin, B \& Miller, K. (2013). Evidence on flipped classrooms is still coming in. Educational Leadership, 70(6), 78-80. Recuperado el 04 de octubre de 2016 desde goo.gl/rqvAjb

Ibáñez, J. S. (2004). Innovación docente y uso de las TIC en la enseñanza universitaria. RUSC. Universities and Knowledge Society Journal, 1(1), 3. Recuperado el 04 de octubre de 2016 desde goo.gl/6rKMgS

Karamustafaoglu, O. (2009). Active learning strategies in physics teaching. Energy Education Science and Tecnology Part B: Social and Educational Studies, 1(1), 27-50. Recuperado el 17 de diciembre de 2016 desde goo.gl/0oT6QR

Lage, M., Platt, G. y Treglia, M. (2000). Inverting the classroom: A gateway to creating an inclusive learning environment. The Journal of Economic Education, 31(1), 30-43. Recuperado el 20 de diciembre de 2016 desde goo.gl/JQgGBE

Lim, C., Kim, S., Lee, J., Kim, H. \& Han, H. (2014). Comparative Case Study on Designing and Applying Flipped Classroom at Universities. International Association for Development of the Information Society. Recuperado el 04 de enero de 2017 desde https://eric.ed.gov/?id=ED557399.

Martínez, W., Esquivel, I. y Castillo, J. M. (2014). Aula invertida o modelo invertido de aprendizaje: Origen, sustento e implicaciones. En Los Modelos Tecno-Educativos, revolucionando el aprendizaje del siglo XXI, 137-154. Recuperado el 05 de diciembre de 2016 desde goo.gl/ uY3sGZ

Merriam, S. B. (2009). Qualitative research. A guide to design and implementation. San Francisco, CA.: Jossey Bass.

Mestre, J. P. (2001). Implications of research on learning for the education of prospective science and physics teachers. Physics Education, 36(1), 44. doi: http://dx.doi.org/0031-9120/01/010044

Observatorio de Innovación Educativa (2014). Aprendizaje invertido. Reporte Edutrends. Monterrey, México: Tecnológico de Monterrey. Recuperado el 05 de diciembre de 2016 desde goo.gl/ Cf8Ywo

Pérez, M., Romero, M. y Romeu, T. (2014). La construcción colaborativa de proyectos como metodología para adquirir competencias digitales. Comunicar: Revista Científica de 
Estudios Pedagógicos XLIII, N 3: 193-204, 2017

AULA INVERTIDA MEDIADA POR EL USO DE PLATAFORMAS VIRTUALES: UN ESTUDIO DE CASO EN LA

FORMACIÓN DE PROFESORES DE FÍSICA

Comunicación y Educación, 21(42), 15-24.doi: http://dx.doi.org/10.3916/C42-2014-01

Pierce, R. \& Fox, J. (2012). Vodcasts and active-learning exercises in a "flipped classroom" model of a renal pharmacotherapy module. Am. J. Pharm. Educ., 76 (10). Recuperado el 10 de noviembre de 2016 desde goo.gl/2dbmlf

Platero, J.,Tejeiro, M. y Reis, F. (2015). La aplicación del Flipped classroom en el curso de dirección estratégica. XII Jornadas Internacionales de Innovación Universitaria pp. 119-133, Universidad Europea de Madrid. Recuperado el 15 de noviembre de 2016 desde goo.gl/vZR8d2

Prensky, M. (2008). Turning on the lights. Educational Leadership, 65(6), 40-45. Recuperado el 04 de noviembre de 2016 desde goo.gl/AIXqHf

Riascos, S. C., Quintero, D. M. y Ávila, G. P. (2009). Las TIC en el aula: percepciones de los profesores universitarios. Educación y educadores, 12(3). Recuperado el 04 de octubre de 2016 desde goo.gl/rP1HUY

Sadaghiani, H. R. (2012). Online prelectures: An alternative to textbook reading assignments. The Physics Teacher, 50 (5), 301-303. doi: http://dx.doi.org/10.1119/1.3703549

Strayer, J. F. (2012). How learning in an inverted classroom influences cooperation, innovation and task orientation. Learning Environments Research, 15, 171-193. doi: http://dx.doi.org/10.1007/ s10984-012-9108-4

Tune, J., Sturek, M. \& Basile, D. (2013). Flipped classroom model improves graduate student performance in cardiovascular, respiratory, and renal physiology. Advances in physiology education, 37(4), 316-320. doi: http://dx.doi.org/10.1152/advan.00091.2013

Vaughan, M. (2014). Flipping the learning: An investigation into the use of flipped classroom model in an introductory teaching course, Education Research and Perspectives, 41, 25-41. Recuperado el 15 de noviembre de 2016 desde goo.gl/Eyku2O

West, R. E. \& Graham, C. R. (2007). Benefits and challenges of using live modeling to help preservice teachers transfer technology integration principles. Journal of Computing in Teacher Education, 23(4), 131-141. Recuperado el 17 de diciembre de 2016 desde goo.gl/RFXEKB

Zainuddin, Z. \& Halili, H. S (2016). Flipped classroom research and trends from different fields of study. International Review of Research in Open and Distributed Learning, 17(3), 313-340. Recuperado el 10 de diciembre de 2016 desde goo.gl/xRB2k3 